\title{
Advanced Processing of Multiples into useful Seismic data for Surface Hydrocarbon Oilfields
}

\author{
Ikwueto O.C. ${ }^{1}$,Ezike I.F. ${ }^{2}$ Ozokwelu N.B. ${ }^{3}$ \\ (Department of Industrial Physics, Anambra State University, Uli, Nigeria $)^{l}$ \\ (S.L.T. Department, Akanulbaim Federal Polytechnic, Unnwana, AfikpoEbonyi State, Nigeria) ${ }^{2}$ \\ (School of Science, Federal College of Education, Asaba, Delta State, Nigeria) ${ }^{3}$
}

\begin{abstract}
Distinguishing multiples from primaries and transforming them into useful signals has been one of the most serious difficulties faced by Geophysicists when processing and interpreting data. Multiples are multiplicative events seen in seismic sections which often seriously impedes correct mapping of the subsurface geology in search of oil and gas reservoirs. These events have undergone more than one reflection. In this paper, we present an approach to transform shot gathers from multiple reflections into primaries by combining a multi-channel weighted cross-correlation (WCC) with surface-related multiple elimination (SRME). The new primaries obtained from the combination exhibit the same kinematics as the original dataset with few transformation artefacts. We also demonstrate that this primaries can accurately estimate missing traces as long as the gaps are within the acquisition spread. While this combination allows the transformation of multiples into primaries for surface seismic data, the latter means that nonlinear seismic imaging can be implemented by a number of linear steps. The result shows that the multiple reflections contain a wealth of information that should be used in seismic processing to improve the resolution of reservoir images beyond current capability.
\end{abstract}

Keywords: Multiple reflection, Surface-Related Multiple Elimination (SRME),Weighted Cross-Correlation (WCC) and Multiple Transformation.

\section{Introduction}

Historically, multiple reflections in seismic data are generally considered to be unwanted noise which are usually being attenuated or removed entirely during the processing of Seismic data, while the primaries are used by the interpreters as the required signal for interpreting the seismic data. Multiples are multiplicative events seen in seismic sections. These events have undergone more than one reflection. They are produced in the data gathering process when the signal doesn't take a direct path from the source to the geologic event and finally back to the receiver on the surface. This causes the signal to arrive back at the receiver at an erroneous time, which, in turn, causes false results and can result in data misinterpretation. There are various kinds of multiples ranging from various long path multiples to short path multiples. Long path multiples appear as separate events in the seismic record, while shortpath multiplesadd tail to the primary reflection. Examples of long path multiples include simple multiples and inter-formational multiples. Short path multiples may be ghosts, near surface multiples, and peg-leg multiples.

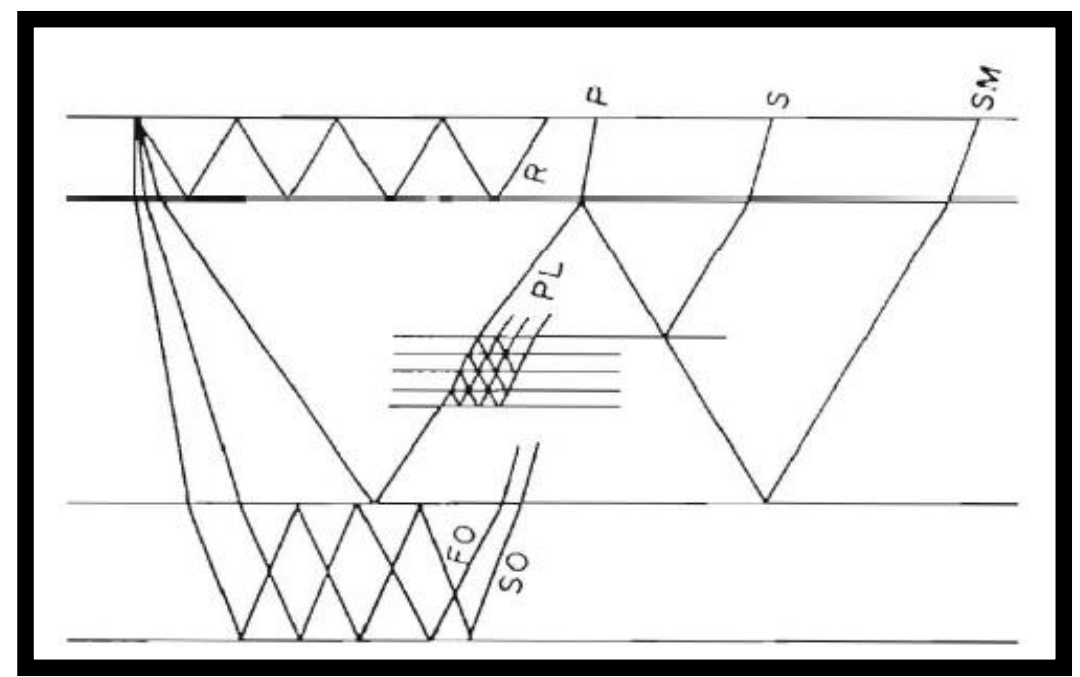

Where:

$P$ - Primary

S - Surface Multiple

SM - Simple Multiple

$\boldsymbol{R}$ - Reverberations

$\boldsymbol{P L}$ - Peg -Leg Multiple S

$\boldsymbol{F O}$ - First Order Multiples

SO - Second Order

Multiples

Fig.1. Various types of multiples.

When only primary reflections are considered for imaging, multiple reflections are usually attenuated as a preprocessing step (Verschuur et al., 1992; Weglein et al., 1997; Guitton, 2003; Sava and Guitton, 2003). However, 
multiples also contain subsurface reflectivity information, and could be treated as signal. For instance, Brown (2004) shows how a joint inversion of both primaries and multiples can provide more knowledge of the earth properties.

There are several techniques of imaging multiples such asKirchoff (Reiter et al., 1991) or crosscorrelogram migration (Sheng, 2001) by transforming the travel time of multiple reflection to that of primary reflections. Multiples can be also imaged by shot-profile migration, considering the primary reflections as a real shot records and the multiple reflections as receiver wave fields (Berkhout and Verschuur, 1994; Guitton, 2002). Instead of being transformed into primaries implicitly, multiples can be explicitly mapped into primaries by cross-correlation (Shan, 2003; Berkhout and Verschuur, 2003) or deconvolution (Shan, 2003).

In this paper, we employed a combination of multi-channel weighted cross-correlation (WCC) and surface-related multiple elimination (SRME) to transform the multiples into useful signals using synthetic data. This method enables us toestablish a resultant primary shot gathers through a cross-correlation of the primary and multiple reflections. These resultant primary shot gathers are then migrated with shot-profile migration. The images obtained from the primaries are then compared to the one of the primaries only. Furthermore, we demonstrated that the estimated primaries are used as focal operator, after which higher-order multiples are transformed to lower-order multiples and first-order multiple become new primaries. Finally, the source characteristics are automatically deconvolved, yielding high-resolution results.

\section{Surface-Related Multiple Elimination (SRME)}

SRME is fully data driven, which means no auxiliary prior information needs to be supplied, such as, velocity and horizon. SRME does not require any subsurface information, nor does it make any assumptions about seismic events such as hyperbolicity or moveout separation between primaries and multiples. As a result, SRME can handle arbitrarily complex multiples (including, in particular, diffracted multiples). In fact, SRME appears to be the only effective approach for attenuating diffracted multiples. SRME performs well at near offsets where the moveout difference is very little.

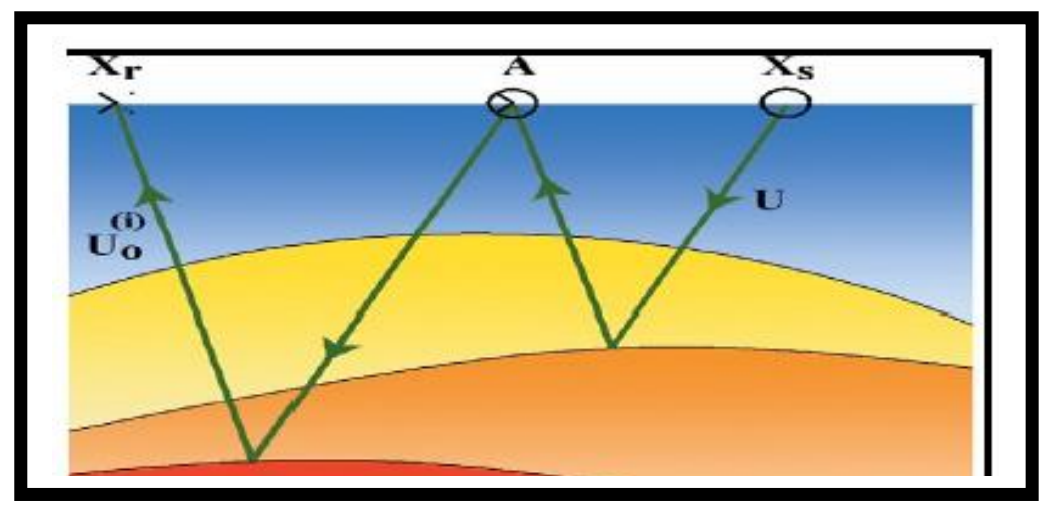

Figure 2: Theory of surface-related multiples (following Berkhout and Verschuur, 1997).

The performance of SRME depends on spatial sampling and signal-to-noise ratio of the input data and robust matching filtering of the predicted multiple models. Unlike in marine case, application of SRME for land data is not a straight away procedure The method has been mainly effective in marine processing in the past due to the reason that marine data is usually better sampled and has higher signal-to-noise ratio than land data. In addition, the water layer provides consistent surface condition to generate multiples. In contrast land data is often poorly sampled in space. In particular neighboring shot lines and receiver lines can be a few hundred meters away from each other. Moreover land data is often contaminated with large amount of random noise and coherent noise (ground roll etc), which can pose a challenge to multiple model prediction. Finally statics caused by uneven surface and non-uniform shallow layers can make it difficult to predict the travel times of multiples. Since SRME predicts the travel time of the multiple using the input data, in the presence of strong velocity variations in the near surface, multiple model predictions might be inaccurate. In addition to this, poor signal to noise ratio and irregular source receiver sampling in the data may limit the effectiveness of the methodology. For optimal results in land data one should address these issues adequately during the preprocessing stage. 


\section{Prediction Of Multiples Using Srme}

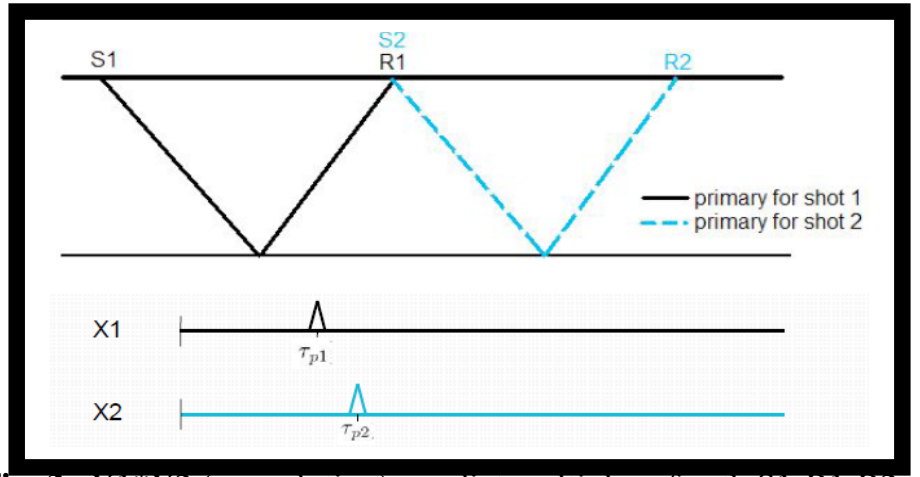

Fig. 3. $\mathrm{X} 1 * \times 2$ (convolution): predict multiples of path S1-RI-R2

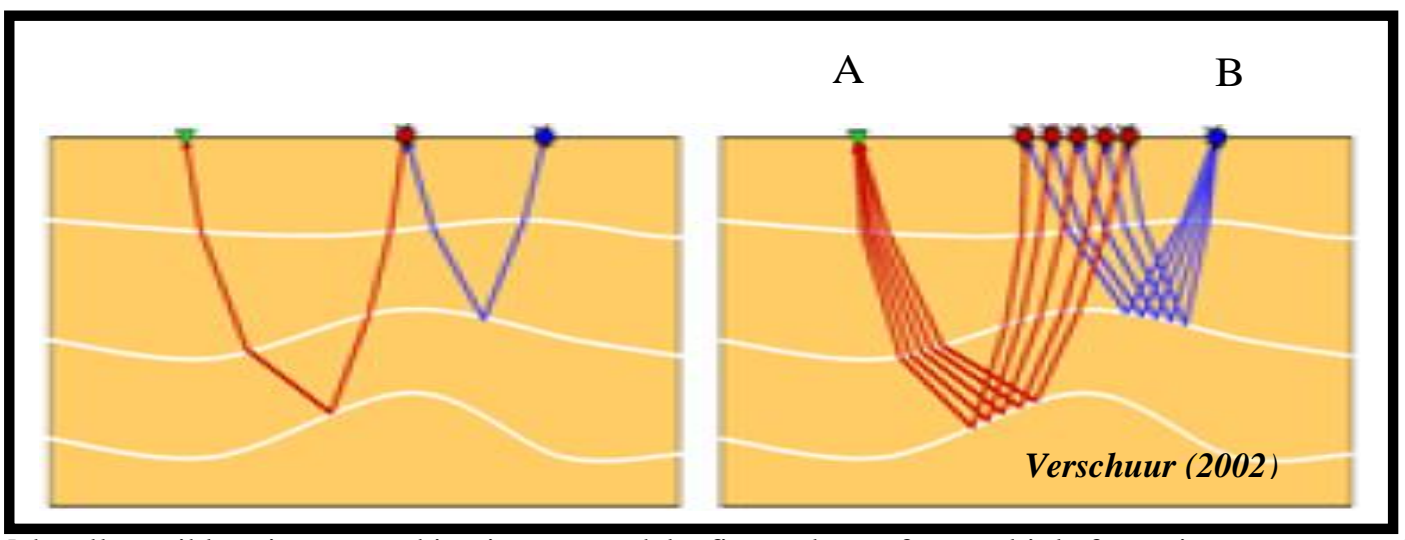

Fig.4. Make all possible primary combinations to model a first order surface multiple for a given source receiver pair. (From D.J. Verschuur, 2002).

Considering the diagram in fig 3 above, in order to understanding and predict the multiples coming from B to A, there must be a convolution of 1) Common short gather (Short at B) and 2) Common receiver gather (receive at B). Hence the sum of all the contribution gives the estimation or the prediction of the multiples.

\subsection{Example with synthetic Data}
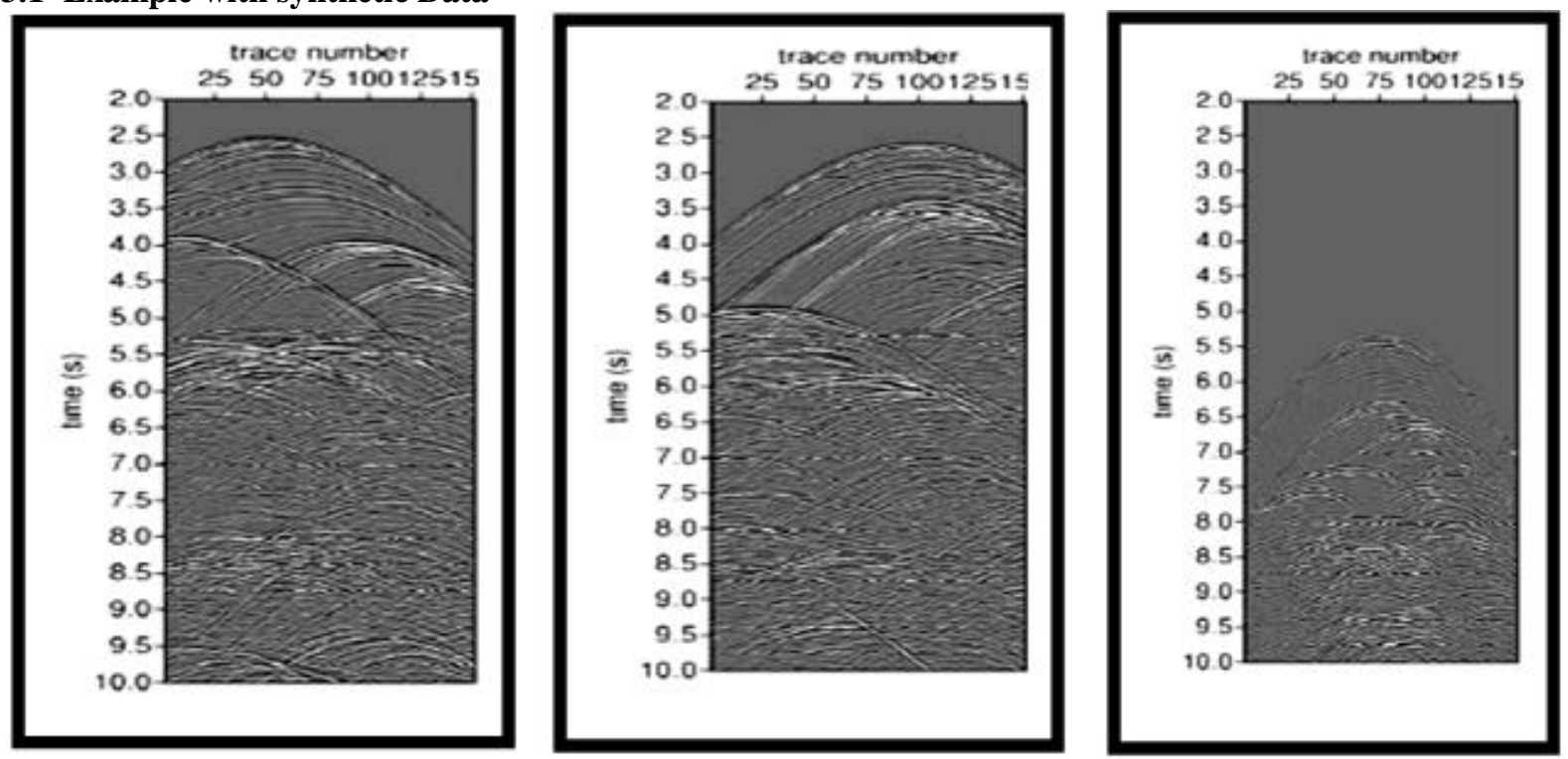

a. Common receiver gather

b. Common short gather

c. Multiple contribution 


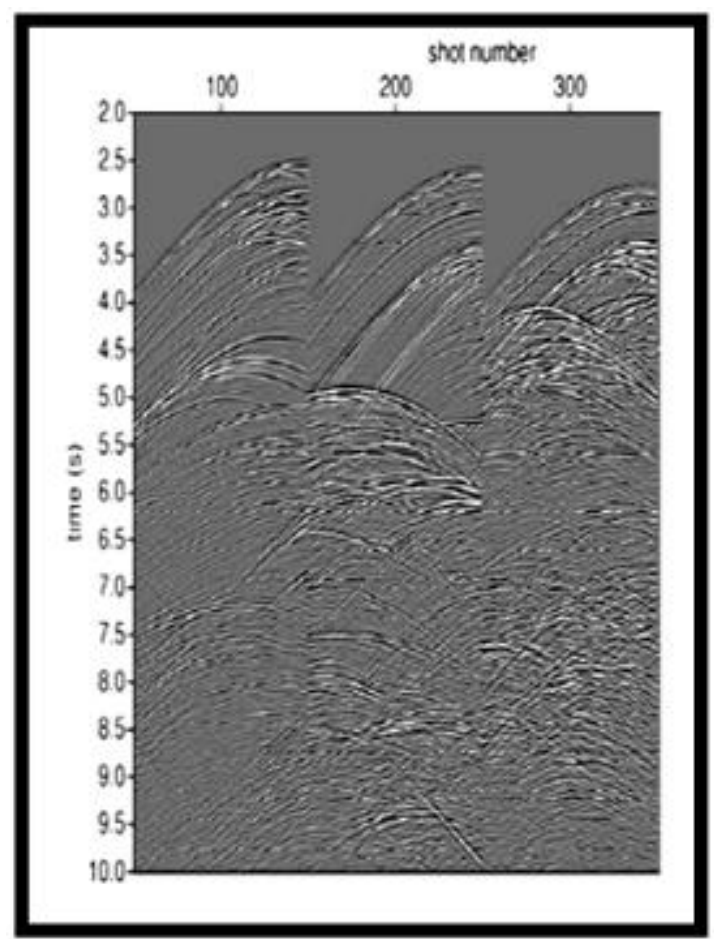

d. Short records with multiples

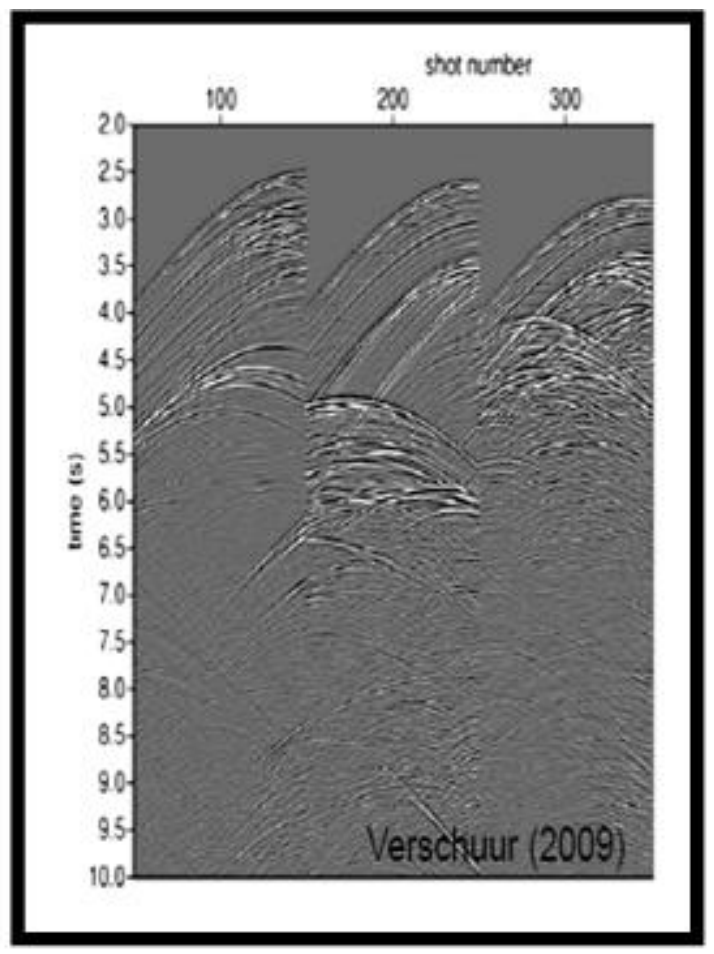

e. Short records after multiple suppression

Fig. 5. Attenuation of multiples using SRME approach: a) Common receiver gather, b) Common short gather, c) Multiple contribution, d) Short records with multiples, e) Short records after multiple

SRME like any other demultiple technique, has its own limitations. However majority of the limitations are applicable to 2D SRME and these include; (1) Finite aperture (2) Directivity effects (3) Spatial aliasing and (4) Cable fathering. In the near offset range SRME works better compared to the far offset range.

\section{Elimination Of The Surface -Related Multiples Using Weighted Crosscorrelation}

To eliminate the surface-related multiples using this method, we show that the relationship between primaries and multiples can also be regarded as a weighted cross-correlation. We also considered the Feedback model in Fig. 5. as shown in the equation [1] below:
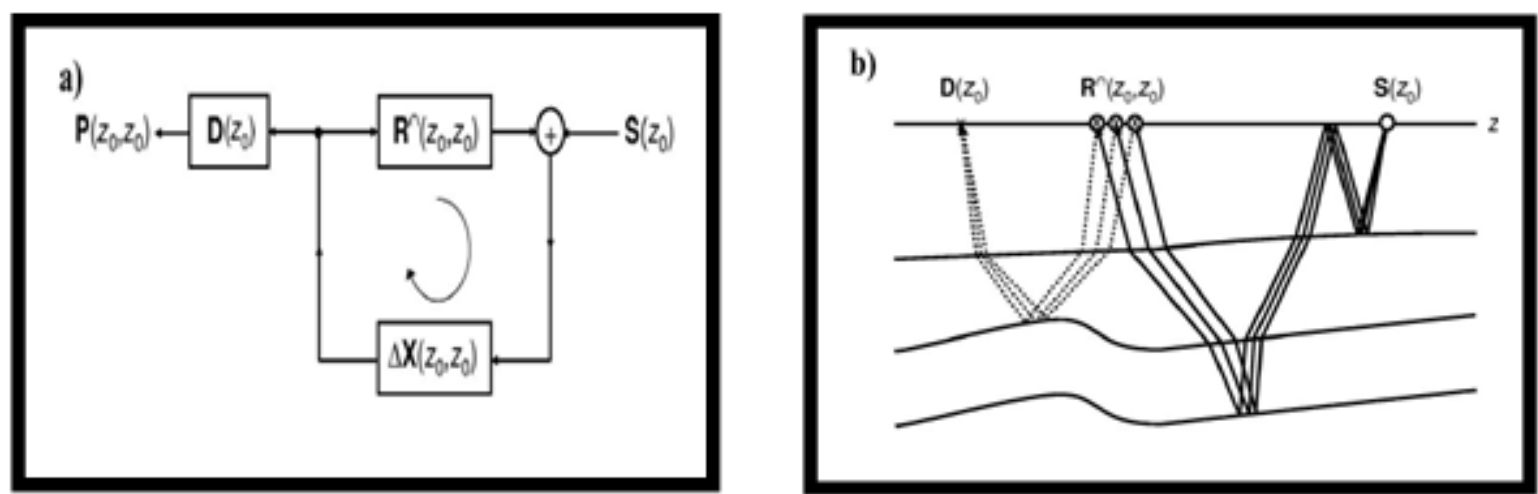

Figure 1. (a) Feedback model for primary reflections (P) and data including surface-related multiples (P), (b) One basic element of the weighted convolution process for surface-related multiples (M), visualized in terms of simple

$\mathbf{P}=\Delta \mathbf{P}+\mathbf{M}=\Delta \mathbf{P}+\Delta \mathbf{P A P}$

Where $\mathbf{P}=$ the data volume with surface-related multiples

$\square \mathbf{P}=$ the data volume without surfac-related multiples

$\mathbf{M}=$ the surface-related multiples.

The expression $\boldsymbol{M}=\boldsymbol{\Delta P} \boldsymbol{A P}$ shows that multiples are predicted by multiplying data matrix $\mathrm{P}$ by

Operator matrix $\square \mathbf{P A}$ 
From equation [1] above, we have:

$\mathbf{P}=\Delta \mathbf{P}+\Delta \mathbf{P A P}$

Solving equation [2] further we have:

$\mathbf{P}=(\mathbf{I}-\Delta \mathbf{P A})^{-\mathbf{1}} \Delta \mathbf{P}$

Applying the series expansion in equation [3] gives:

$\mathbf{P}=\Delta \mathbf{P}+(\Delta \mathbf{P A}) \Delta \mathbf{P}+(\Delta \mathbf{P A})^{2} \Delta \mathbf{P}+(\Delta \mathbf{P A})^{3} \Delta \mathbf{P}+\cdots$,

Where $\Delta$ P represents primaries, ( $\square \mathbf{P A}) \square \mathbf{P}$ represents first-order multiples, and ( $\square \mathbf{P A})^{2} \square \mathbf{P}$ represents secondorder multiples, etc. Equation [4] shows that multiplying P by the weighted primary operator ( $\square$ PA) transforms primaries into first-order multiples and first-order multiples into second-order multiples, etc.:

$\mathbf{M}=\Delta \mathbf{P A P}=(\Delta \mathbf{P A}) \Delta \mathbf{P}+(\Delta \mathbf{P A})^{2} \Delta \mathbf{P}+(\Delta \mathbf{P A})^{3} \Delta \mathbf{P} \ldots+$

Let us use the operator $\Delta \mathrm{P}$ in a correlation mode to define a focal transform (Berkhout and Verschuur, 2003; Berkhout et al., 2004):

$\mathbf{Q}=\mathbf{F P}$ (Forward focal transform)

And

$\dot{P}=\mathbf{G Q}$ (Inverse focal transform)

ButG $=\Delta \mathbf{P}$

Also

$\mathbf{F}=\Delta \mathbf{P}^{-1} \approx \Delta \mathbf{P}^{H} B$

Where $\mathbf{B}=\left(\Delta \mathbf{P} \Delta \mathbf{P}^{H}+\epsilon^{2} I\right)^{-1}$

(least-squares version)

$\mathbf{H}=$ Hermitian operator

$\epsilon^{2}=$ A small positive constant that we use

The forward focal transformation implies taking the seismic data with multiples to the focal domain using a multidimensional deconvolution (or WCC) with an estimate of the primary reflections. Inverse focal transform implies moving from the focal domain back to the original data domain. Application of the operator $\mathrm{F}$ will transform the primary reflections into a band-limited focal point, first-order reflections into primaries, and second-order reflections into first-order reflections, etc.:

In order to obtain the equation for weighted focal area, we considered the following equations below:

for stabilization

$$
\mathbf{Q}=\Delta \mathbf{P}^{-1} \mathbf{P} \approx \Delta \mathbf{P}^{\mathrm{H}} \mathbf{B P}
$$

$=\hat{\mathbf{I}}+\hat{\mathbf{A}} \Delta \mathbf{P}+\hat{\mathbf{A}}(\boldsymbol{\Delta} \mathbf{P A}) \Delta \mathbf{P}+\hat{\mathbf{A}}(\Delta \mathbf{P A})^{2} \Delta \mathbf{P} \ldots$

$\mathbf{Q}=\hat{\mathbf{I}}+\hat{\mathbf{A}} \mathbf{P}$,

But $\hat{I}=\Delta \mathbf{P}^{-1} \Delta \mathbf{P}($ focal area $)$

Also, $\hat{\mathbf{A}}=\mathbf{I} \mathbf{A}$ (Weighted focal Area)

Where $\Delta \mathbf{P}^{\mathrm{H}} \mathbf{B P}=$ weighted cross-correlation,

$\mathbf{B}=$ weighting factors

In weighted Cross-correlation method, all shot records are involved in computing one output trace. The presence of $\Delta \mathbf{P}^{\mathrm{H}} \mathbf{B P}$ in the above equation is remove one round trip through the subsurface, while Matrix $\mathbf{P}$ represents the total response that has been recovered from the multiples. The above equations (11 - 15) shows the theoretical base for a new concept in handling multiple scattering that involves separating primaries from multiples and transforming multiple reflections into primaries. 


\section{Methodology}

The mapping of multiples into primaries such that the newly obtained primaries also can be used in imaging algorithms is one of the applications of focal transformation. Once multiples are transformed into primaries, all other processing steps that follow are the same as in traditional primary processing. This avoids the need to reformulate imaging algorithms for multiple scattering. Such was described by BerkhoutandVerschuur (1995) and further illustrated by Guitton (2002).

In other to demonstrate the transformation of multiples into primaries we replace convolution with correlation using data from one reflector medium. Hence, we apply the estimated primaries in equation [9] to the data as focal operator as shown below:

$\mathbf{F}=\Delta \mathbf{P}^{-1}$

Applying equation [16] to the data in equation [2] and equation [5] as shown below:

$\mathbf{P}=\Delta \mathbf{P}+\Delta \mathbf{P A P}$ And $\mathbf{M}=\Delta \mathbf{P A P}$

This yield focal transform:

$\mathbf{Q}=\mathbf{F P}=\mathbf{I}+\mathbf{A P}=\mathbf{I}+\mathbf{X}$

Here are some synthetic examples of the SRME and WCC Transforms in action, which show how the combination is being used to transform the multiples from the multiple-zone into primaries and the results obtained.

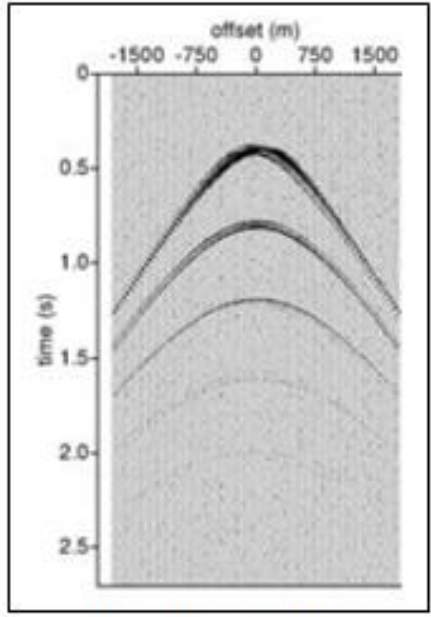

a. Shot with multiples, $P$

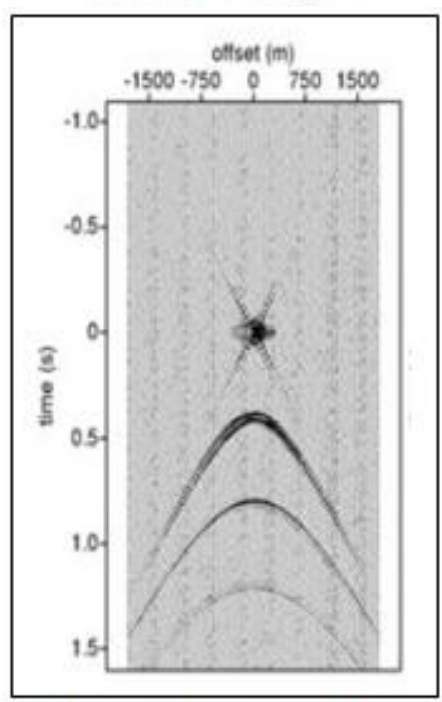

d. Focal transform, $Q$

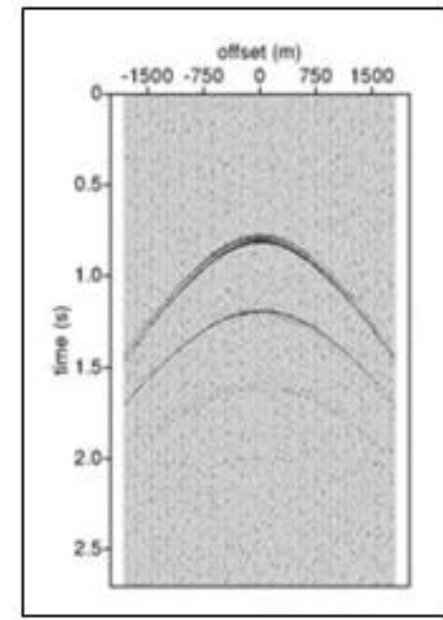

b. Predicted multiples, $M$

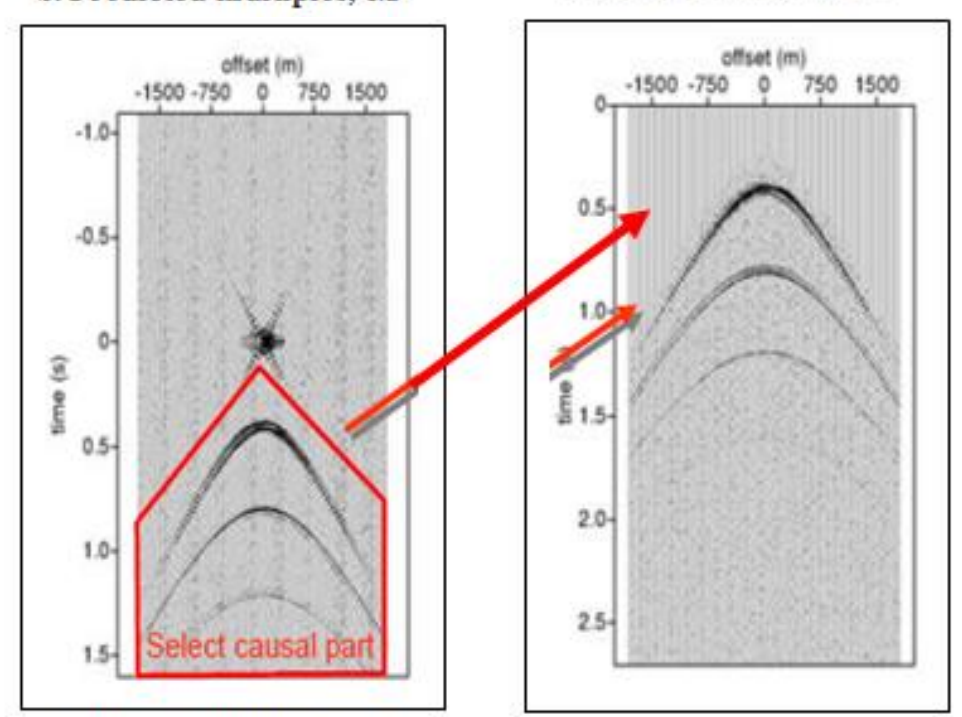

e. Muted focal transform

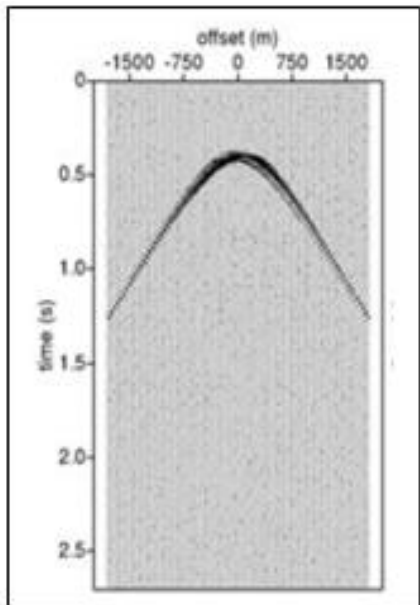

c. Primaries SRME, $\triangle \mathrm{P}$

f. New primaries from

Fig. 5. Transformation of multiple reflection into primaries: synthetic data example a.) Shot with multiples, $P$, b.) Predicted multiples, M, c.) Primaries SRME, $\Delta \mathrm{P}$, d.) Focal transform, Q, e.) Muted focal transform, f.) New primaries from multiples 
After SRME, the estimated primaries are used as focal operator, after which higher-order multiples are transformed to lower-order multiples and first-order multiple become new primaries. Note also that the source characteristics are automatically deconvolved, yielding high-resolution results (Image of the subsurface).

\section{Discussion}

Fig. 5. Above shows how the transformation of multiples into primaries (WCC) is being used to construct primaries at missing offsets from multiple scattering at available offsets. We expect that diffracted multiple reflections contain a wealth of information to fill in primary information that has not been recorded.

By transforming shot records into common-focus-point (CFP) gathers, sources at the surface are repositioned in the subsurface (Berkhout, 1997). This implies that ifshot records is replaced with CFP gathers, the theory presented also is applicable to seismic responses at the surface that come from sources in the subsurface. We also observed that such reformulation in terms of CFP gathers still requires a focal transform operator with both sources and detectors at the surface:

The result shows that the active seismic measurements (i.e., recordings from man-made sources at the surface) are required to transform multiples into primaries from passive seismic measurements (i.e., recordings from natural sources in the subsurface) when these sources are not fully uncorrelated (see, Wapenaar et al.2004)

\section{Conclusion}

In this work, the combination of SRME and WCC was discussed in which 1D SRME application has given satisfactory results. Long period surface multiples were transformed into primaries successfully. The use of weighted cross-correlation (WCC) technique developed by (D.J. Verschuur and A.J. Berkhout) based on the SRME principles resolves this issue of multiple transformation successfully by two modifications: 1) an estimate of the primary reflection data is used as the correlation operator and is applied to the surface data with multiples and 2) the cross-correlation process is replaced by a multidimensional inversion procedure, which is implemented as a weighted cross-correlation. The resultant cleaner seismic data will consequently generate better imaging for interpretation, especially for complex oilfields.

\section{References}

[1]. Poli N. et al., Surface Related Multiple Elimination: A Case study from East Coast India, Proc. 10th Biennial International Conference \& Exposition,Dehradun, India, 2013, 217 - 220.

[2]. Berkhout A. J. \&Verschuur D.J, Imaging of multiple reflections (published online, 2006) Geophysicis 17(4), 209-220.

[3]. Shan G and GuittonA., 2004, Migration of surface-related multiples: tests on the Sigsbee2B dataset, SEG Annual Meeting, Denver, Colorado. $1285-1288$

[4]. Berkhout, A., and Verschuur, D., 2003, Transformation of multiples into primary reflections in 73rd Ann. Internat. Mtg. Soc. Of Expl. Geophys. 1925.1928.

[5]. Hargreaves N., (2006), Surface multiple attenuation in shallow water and the construction of primaries from multiples, SEG New Orleans Annual Meeting, 2689 - 2693 